Jeffrey F. Barletta · Gina-Marie Barletta •

Patrick D. Brophy • Norma J. Maxvold •

Richard M. Hackbarth · Timothy E. Bunchman

\title{
Medication errors and patient complications with continuous renal replacement therapy
}

Received: 14 September 2005 / Revised: 2 November 2005 / Accepted: 1 December 2005

(C) IPNA 2006

\begin{abstract}
Continuous renal replacement therapy (CRRT) is commonly used for renal support in the intensive care unit. While the risk of medication errors in the intensive care unit has been described, errors related specifically to CRRT are unknown. The purpose of this study is to characterize medication errors related to CRRT and compare medication errors that occur with manually compounded solutions versus commercially available solutions. We surveyed three separate internet-based, pediatric list serves that are commonly used for communications for programs utilizing CRRT. Data regarding CRRT practices and medication errors were recorded. Medication errors were graded for degree of severity and compared between programs using manually compounded dialysis solutions versus commercially available dialysis solutions. In a survey with 31 program responses, 18 reported medication errors. Two of the 18 were related to heparin compounding, while $16 / 18$ were due to solution compounding errors. Half of the medication errors were
\end{abstract}

Timothy E. Bunchman is the PI for the Peds CRRT list serve and a consultant for Dialysis Solutions, Inc. He has received grant monies via the registry from Gambro, Baxter, Dialysis Solutions, Inc., B. Braun and grant monies from the Michigan Chapter of the National Kidney Foundation.

J. F. Barletta $(\bowtie)$

Department of Pharmacy, Spectrum Health,

100 Michigan St NE (MC001),

Grand Rapids, MI 49503 USA

e-mail: Jeffrey.Barletta@spectrum-health.org

Tel.: +1-616-3912578

Fax: +1-616-3919598

G.-M. Barletta · T. E. Bunchman

Pediatric Nephrology \& Transplantation,

DeVos Children's Hospital,

Grand Rapids, MI, USA

P. D. Brophy

Pediatric Nephrology, University of Michigan,

Ann Arbor, MI, USA

N. J. Maxvold · R. M. Hackbarth

Pediatric Critical Care, DeVos Children's Hospital,

Grand Rapids, MI, USA classified as causing harm, two of which were fatal. All medication errors were reported by programs that manually compounded their dialysis solutions. Medication errors related to CRRT are associated with a high degree of severity, including death. Industry-based, commercially available solutions can decrease the occurrence of medication errors due to CRRT.

Keywords Medication errors - Continuous - Dialysis · Dialysis solutions

\section{Introduction}

Continuous renal replacement therapy (CRRT) was first introduced in 1977 for fluid overload in the intensive care unit and has now become commonplace in the critical care setting [1, 2]. CRRT encompasses a variety of techniques with clearance mechanisms that utilize a convective mode [(continuous veno-venous hemofiltration $(\mathrm{CVVH})]$, a diffusive mode [(continuous veno-venous hemofiltration with dialysis (CVVHD)] or a combination [(continuous veno-venous hemodiafiltration (CVVHDF)] [3]. All CRRT modalities require either replacement solutions (for convection) or dialysis solutions (for diffusion) [4]. Typically, these solutions have been bicarbonate based, as data have shown that bicarbonate-based solutions improve hemodynamic stability and outcome in patients compared to lactate-based solutions [5]. Prior to 2000, however, no Food and Drug Administration (FDA)-approved bicarbonate solutions were available in the United States of America; therefore, many programs began using pharmacy made solutions that were compounded manually.

There is considerable risk for medication errors when a high volume of dialysis solutions are compounded manually. For example, a single patient may require up to 501 of dialysis solutions per day. This leads to a substantial increase in workload on behalf of the pharmacy, with the average time to prepare one 31 bag being approximately 20-30 min. Since these solutions are not routinely assayed and verified for the proper concentration, 
many errors go unrecognized. Programs have privately reported medication errors related to dialysis solutions for CRRT, yet published data, until recently, have been nonexistent [6].

Currently, there are three industry-developed, bicarbonate-based solutions that are FDA approved: Normocarb (Dialysis Solutions, Richmond Hills, Ontario, Canada), Prismasate (Gambro, Lakewood, Calif., USA) and Accusol (Baxter, Deerfield, Ill., USA). It is presumed that these standardized, pre-mixed solutions will offer a tremendous advantage with regard to medication safety.

The purpose of this study is to characterize medication errors related to dialysis solutions used for CRRT. Secondly, medication errors will be compared between pharmacy-made/manually compounded solutions and industry-based/commercially available solutions.

\section{Materials and methods}

Internet-based list serves were used to capture data related to medication errors and CRRT. Three individual list serves were queried, which included pediatric critical care [i.e., PedsCCM (http://pedsccm.wustl.edu/PedsCCM Home page.html)], pediatric nephrology [i.e., PEDNEPH (http:// listhost.uchicago.edu/mailman/listinfo/pedneph)] and pediatric CRRT [i.e., PEDCRRT (http://listhost.uchicago. edu/mailman/listinfo/pedcrrt)]. Those list serves are made up of over 1,000 clinicians and allow for communication among programs, both publicly and privately, as well as open discussion between critical care physicians, nephrologists, nurses, and pharmacists. Overall, there are approximately 100 to 120 individual programs within the members of these list serves. Information requested included the CRRT modality used (convective vs diffusive clearance), the type of anticoagulation (heparin vs citrate), the type of solutions used (manually compounded vs commercially available), as well as complications related to any of the above. In addition, programs were asked if they did identify errors, what steps were taken to correct the problems. All respondents were asked to return comments privately and assured that all communication would be destroyed and that the data would be tabulated without there being any ability to link back to the primary source. The principle investigator of this study (T.E.B.) reviewed all data and ensured that there were no duplications within programs.

The severity of medication errors was categorized by criteria developed by the National Coordinating Council for Medication Error Reporting and Prevention [7]. This system uses three major categories of errors (i.e., no harm, harm, death), with various subcategories under each division (Table 1).

Descriptive statistics were used, as most data were not amenable to inferential testing. To compare medication errors between manually compounded and industry-based dialysis solutions we used, as appropriate, either Pearson's chi square test or Fisher's exact test. A $P$ value $<0.05$ was
Table 1 Categories of medication error severity (adapted from [7])

\begin{tabular}{|c|c|c|}
\hline $\begin{array}{l}\text { Major } \\
\text { division }\end{array}$ & Subcategory & Description \\
\hline \multirow[t]{4}{*}{$\begin{array}{l}\text { Error, } \\
\text { no harm }\end{array}$} & Category B & Error, but medication did not reach patient \\
\hline & Category C & Error reaches patient, but no harm \\
\hline & & Error reaches patient, but not administered \\
\hline & Category D & $\begin{array}{l}\text { Error occurred, resulted in increased patient } \\
\text { monitoring, but no harm to patient }\end{array}$ \\
\hline \multirow[t]{4}{*}{$\begin{array}{l}\text { Error, } \\
\text { harm }\end{array}$} & Category E & $\begin{array}{l}\text { Resulted in need for therapy or intervention, } \\
\text { caused temporary harm }\end{array}$ \\
\hline & Category F & $\begin{array}{l}\text { Resulted in initial or prolonged hospital- } \\
\text { ization and temporary harm to patient }\end{array}$ \\
\hline & Category G & Resulted in permanent harm \\
\hline & Category H & $\begin{array}{l}\text { Resulted in near-death event } \\
\text { (anaphylaxis, cardiac arrest) }\end{array}$ \\
\hline $\begin{array}{l}\text { Error, } \\
\text { death }\end{array}$ & Category I & \\
\hline
\end{tabular}

considered statistically significant. SPSS, version 13.0 (Chicago, Ill., USA) was used for all analyses.

\section{Results}

Thirty-one programs responded to the question. Patient volumes of CRRT per year ranged from two to 41 . Modalities included CVVH (nine programs), CVVHD (seven programs), CVVHDF (seven programs), and varying CRRT modalities (eight programs). The anticoagulant used was heparin in 20 programs and citrate in 11 programs. Of the 20 programs that used diffusive methods, 13 utilized manually compounded, bicarbonate-based solutions, while seven programs utilized commercially available, industry-based solutions (bicarbonate-based Normocarb in two programs, bicarbonate-based Prismasate in two programs, and lactate-based Baxter hemofiltration solution in three programs). Of the 20 programs that used convective clearance methods, 14 utilized manually compounded, bicarbonate-based solutions, two utilized industry-developed, bicarbonate-based (Normocarb) and four utilized normal saline or lactate Ringer's solutions.

Of the 31 programs, $18(58 \%)$ noted at least one error. Table 2 lists the errors categorized according to severity. Two errors involved anticoagulation, and 16 were due to solution compounding errors. In those programs that had anticoagulation errors, both were related to improper mixing of heparin solutions, resulting in over-heparinization of the patients. Both of these resulted in non-life threatening, mild bleeding. The remaining 16 errors involved compounding errors, all of which were reported by programs using manually compounded solutions: seven used for replacement and nine used for dialysate. There were no errors reported by programs using industrymade solutions (Table 3). The patient complications associated with these errors included six seizures related to either hypo- or hypernatremia, one cardiac arrest (with 
survival) and two fatalities. Both fatalities were related to inappropriate potassium concentrations, while the cardiac arrest was related to calcium. Seven patients had no complications.

Programs that reported errors implemented changes in order to avoid future risk. Of those reviewed, 13 programs changed from pharmacy-made, manually compounded, bicarbonate-based solutions to commercially available, industry-developed, bicarbonate-based solutions for either replacement or dialysate solution (11 Normocarb, and two Prismasate). Two programs purchased automated solution compounders to be used specifically for large-volume parenteral solutions, ten programs began having nursing staff double check the labels of all bags, and many programs implemented pre-printed orders to minimize handwriting legibility errors. Three programs initiated the practice of confirming the composition of each solution by laboratory analysis. Interestingly, programs that utilized chemical analysis of each dialysate or replacement solution reported a delay of up to $45 \mathrm{~min}$ for delivery of the solution to the bedside, compared to the previous time that solutions were available.

\section{Discussion}

Medication dosing and drug preparation in pediatrics requires multiple considerations and thus can be time consuming and error prone [8-11]. Despite the high recognition of medication safety, the prevention of medication errors continues to be a major undertaking for clinicians in healthcare today. While various methods exist to detect medication errors, it is difficult to quantify and/or catch errors related to dialysis solutions, since most are related to compounding/mixing the solution itself. Since few institutions chemically test solutions for the proper additives, many errors go unrecognized. In fact, only a single report exists describing a compounding error that resulted in excessive potassium in CRRT solutions, ultimately leading to the deaths of two patients [6]. Reports of medication errors in pediatric CRRT have been discussed at national meetings but have never been quantified. This lack of quantification has not been helpful in supporting the need for industry-made solutions for CRRT.

Recent reports have described the incidence of medication errors in the intensive care unit (ICU). In one study of error-prone medications, the percentage of errors classified

Table 2 Severity of medication errors

\begin{tabular}{lll}
\hline Major division & Subcategory & Number of errors (\%) \\
\hline Error, no harm & Category C & $7(39 \%)$ \\
& Category D & $2(11 \%)$ \\
Error, harm & Category F & $6(33 \%)$ \\
& Category H & $1(6 \%)$ \\
Error, death & Category I & $2(11 \%)$ \\
Total errors & & 18 \\
\hline
\end{tabular}

Table 3 Medication errors and solution type

\begin{tabular}{lccr}
\hline Solution & \multicolumn{2}{l}{ Number of errors } & $P$ \\
\cline { 2 - 3 } & Manually Compounded Industry Developed & \\
\hline Dialysate & $9 / 13$ & $0 / 7$ & 0.005 \\
Replacement & $7 / 14$ & $0 / 6$ & 0.051 \\
Total & $16 / 27$ & $0 / 13$ & $<0.001$ \\
\hline
\end{tabular}

The data are reported as number of errors/number of programs

as "causing harm" was $1 \%$ [12]. The drug classes associated with the most errors were vasoactive medications $(33 \%)$ followed by sedative/analgesics $(26 \%)$. In a second study, $11 \%$ of serious errors were considered life threatening [13]. Most errors (61\%) occurred during the ordering or execution of treatment. We noted that half of the reported errors specific to CRRT would be classified as causing harm. Furthermore, over half of the respondents of this survey have reported medication errors related to CRRT solutions. Although it is difficult to comment on the true incidence of medication errors related to CRRT, when they do occur, they can be associated with significant adverse clinical outcomes.

A majority of the medication errors reported were related to compounding or mixing the dialysis solutions. Most institutions still compound their dialysis solutions by manually adding each individual electrolyte, despite there being a number of industry-based solutions commercially available. In 2000, Normocarb was the first bicarbonatebased solution made available for CRRT. This is now being used for dialysis as well as replacement fluid $[5,14,15]$. In 2002, Gambro introduced their product, Prismasate, which is available for dialysate. Finally, in 2004, Baxter developed their bicarbonate-based solution, Accusol.

The availability of these industry-made solutions offers tremendous advantages in terms of efficiency and patient safety. It is pertinent that clinicians recognize their availability. The economic impact of these solutions has not been determined, but factors that must be considered are the cost and time devoted to preparation, along with the cost of a medication error. While the acquisition cost of these solutions may be higher than the cost of the constituents used to manually compound dialysis solutions, the overall cost to the system may be lower when the consequences of an error are included. We encourage programs that are using extemporaneously made solutions to consider the use of commercially available solutions to minimize serious and potentially lethal complications associated with solution compounding in CRRT.

While most medication errors were related to dialysis solutions, two involved anticoagulation. Anticoagulation protocols for CRRT are either heparin based or citrate based. Medication errors with heparin are common in the ICU and may, in part, be due to the varying concentrations in which it can be prepared. Citrate is available in premixed bags as either trisodium citrate or anticoagulant citrate dextrose solution formula A (ACD-A) [5, 14-16]. The concentrations of citrate and sodium are different in the two solutions and, at first glance, may appear similar. The 
similar appearance allows for the potential of a substitution error or a sound-alike/look-alike error. Institutions are encouraged to implement safeguards to prevent this from occurring or, ideally, to have only one product available on formulary.

Limitations of our study include the inability to report a true incidence of medication errors related to CRRT, due to the manner in which programs were queried. Regardless of the incidence, however, clinicians must recognize that serious medication errors can occur with CRRT. Secondly, medication errors could have occurred that were not clinically significant and therefore not recognized. Finally, the number of institutions that did respond to this query may not be representative of the entire CRRT community.

\section{Conclusion}

In conclusion, medication errors related to CRRT solutions are associated with a high degree of severity, including death. The available industry-made bicarbonate-based solutions are safe and are a reasonable alternative to manually compounded solutions. These commercially available solutions facilitate efficient patient care, markedly reduce the risk of compounding error, and may reduce the liability for the institution.

\section{References}

1. Bunchman TE, McBryde KD, Mottes TE, Gardner JJ, Maxvold NJ, Brophy PD (2001) Pediatric acute renal failure: outcome by modality and disease. Pediatr Nephrol 16:1067-1071

2. Burchardi H (1998) History and development of continuous renal replacement techniques. Kidney Int 53 [Suppl 66]: S12-S124

3. Palevsky PM, Bunchman TE, Tetta C (2002) The Acute Dialysis Quality Initiative-Part V: operational characteristics of CRRT. Adv Ren Replace Ther 9:268-272
4. Kellum JA, Johnson JP, Kramer D, Palevsky P, Brady JJ, Pinsky MR (1998) Diffusive vs convective therapy: effects on mediators of inflammation in patient with severe systemic inflammatory response syndrome. Crit Care Med 26:1995-2000

5. Barenbrock M, Hausberg M, Matzkies F, de la Motte S, Schaefer RM (2000) Effects of bicarbonate- and lactatebuffered replacement fluids on cardiovascular outcome in CVVH patients. Kidney Int 58:1751-1757

6. New Canadian guidelines released over potassium chloridelinked products and CRRT solutions after ESRD patient deaths (2004). Nephrology News \& Issues 18, p 12

7. The National Coordination Council for Medication Error Reporting and Prevention (1996) New release: medication error council promotes categorization index. September 4. http://www.nccmerp.org/medErrorCatIndex.html

8. Daschner M (2005) Drug dosage in children with reduced renal function. Pediatr Nephrol 20:1675-1686

9. Vidal L, Shavit M, Fraser A, Paul M, Leibovici L (2005) Systematic comparison of four sources of drug information regarding adjustment of dose for renal function. BMJ 331:263

10. van Rossum LK, Mathot RA, Cransberg K, Zietse R, Vulto AG (2005) Estimation of the glomerular filtration rate in children: which algorithm should be used? Pediatr Nephrol 20:1769-1775

11. Filler G, Lepage N (2003) Should the Schwartz formula for estimation of GFR be replaced by cystatin C formula? Pediatr Nephrol 18:981-985

12. Calabrese AD, Erstad BL, Brandl K, Barletta JF, Kane SL, Sherman DS (2001) Medication administration errors in adult patients in the ICU. Intensive Care Med 27:1592-1598

13. Rothschild JM, Landrigan CP, Cronin JW, Kaushal R, Lockley SW, Budick E, Stone PH, Lilly CM, Katz JT, Czeisler CA, Bates DW (2005) The critical care safety study: the incidence and nature of adverse events and serious medical errors in intensive care. Crit Care Med 33:1694-1700

14. Bunchman TE, Maxvold NJ, Brophy PD (2003) Pediatric convective hemofiltration (CVVH): normocarb replacement fluid and citrate anticoagulation. Am J Kidney Dis 42: $1248-1252$

15. Tobe SW, Aujla P, Walele AA, Oliver MJ, Naimark DM, Perkins NJ, Beardsall M (2003) A novel regional citrate anticoagulation protocol for CRRT using only commercially available solutions. J Crit Care 18:121-129

16. Mehta RL, McDonald BR, Aguilar MM, Ward DM (1990) Regional citrate anticoagulation for continuous arteriovenous hemodialysis in critically ill patients. Kidney Int 38:976-981 